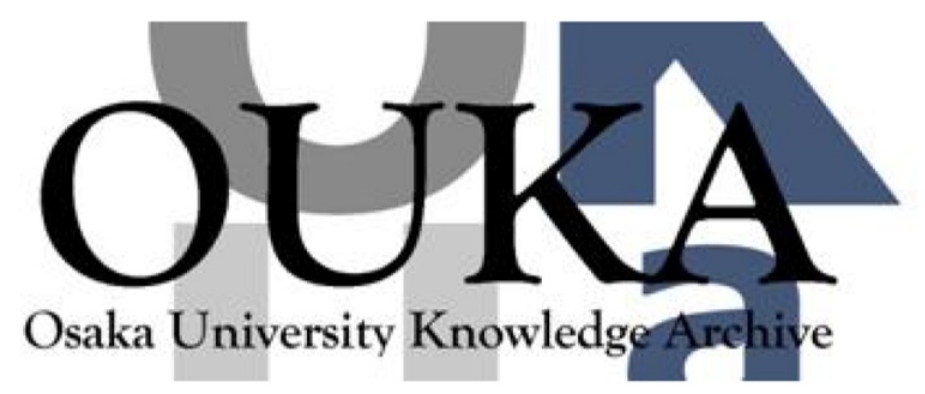

\begin{tabular}{|c|l|}
\hline Title & $\begin{array}{l}\text { Phonon propagation in isotopic diamond } \\
\text { superlattices }\end{array}$ \\
\hline Author(s) & Weng, H. K.; Nagakubo, A.; Ogi, H. et al. \\
\hline Citation & Physical Review B. 104(5) p. 054112-1-p. 054112-7 \\
\hline Issue Date & $2021-08-16$ \\
\hline oaire:version & VoR \\
\hline URL & https://hdl. handle.net/11094/84175 \\
\hline rights & Copyright 2021 by the American Physical Society \\
\hline Note & \\
\hline
\end{tabular}

Osaka University Knowledge Archive : OUKA

https://ir. Library. osaka-u. ac. jp/

0saka University 


\title{
Phonon propagation in isotopic diamond superlattices
}

\author{
H. K. Weng, A. Nagakubo, and H. Ogi $\odot^{*}$ \\ Graduate School of Engineering, Osaka University, 2-1 Yamadaoka, Suita, Osaka 565-0871, Japan \\ H. Watanabe \\ National Institute of Advanced Industrial Science and Technology, Tsukuba, Ibaraki 305-8568, Japan
}

(Received 3 June 2021; revised 15 July 2021; accepted 3 August 2021; published 16 August 2021)

\begin{abstract}
The out-of-plane thermal conductivity and elastic constant of epitaxial $[100]{ }^{12} \mathrm{C} /{ }^{13} \mathrm{C}$ superlattice diamonds with layer thicknesses of 1,30 , and $100 \mathrm{~nm}$ are evaluated by picosecond ultrasound spectroscopy. The measured elastic constants of the superlattices are equivalent to those of single-layer diamond thin films. This result confirms our success in synthesizing superlattice specimens with few defects at the interfaces. Therefore, the phonon transport behavior is governed by the mass difference, not the interfacial defects. The measured thermal conductivity of the superlattices is lower than that of a pure ${ }^{12} \mathrm{C}$ isotope diamond thin film. We estimated the lattice thermal conductivity using the lattice dynamics calculation, attributing the lowered thermal conductivity to the decrease in the phonon group velocity in superlattices. We further consider the effect of mini-umklapp scattering in the superlattice, which explains the dependence of the thermal conductivity on the layer thickness. We reveal that the mini-umklapp scattering effect becomes significant only for an isotope diamond superlattice because of the high Debye temperature and large relative mass difference.
\end{abstract}

DOI: 10.1103/PhysRevB.104.054112

\section{INTRODUCTION}

Recently, superlattices have been intensively studied because they exhibit special physical properties in various fields, especially electronics, optoelectronics, and magnetic fields. For instance, $\mathrm{Si}_{x} / \mathrm{Ge}_{1-x}$ and $\mathrm{GaAs} / \mathrm{Al}_{x} \mathrm{Ga}_{1-x}$ As superlattices demonstrate the formation of a unique quantum well [1-3], and $\mathrm{Fe} / \mathrm{Cr}$ superlattices show the giant magnetoresistance [4]. The thermal conduction of superlattices has been developed as a research area [5-7]. However, a noticeable bottleneck is the direct comparison of the measured and calculated thermal conductivities for a superlattice thin film because of the interfacial defects. Defects at superlattice interfaces, including the interfacial dislocations, local disbonding, and weakened layer bonds, are mainly caused by the lattice misfit, and they significantly decrease the out-of-plane elastic constant [8-10]. Because these defects also affect the out-of-plane thermal conductivity, comparing the measurement and theory is difficult. The thermal conductivity of the superlattice decreases with a decrease in the layer period because of phonon scattering due to the defects from lattice mismatch. Lee et al. [5] used the $3 \omega$ method and reported that the thermal conductivity of the $\mathrm{Si} / \mathrm{Ge}$ superlattice was more than one order of magnitude lower than the bulk value. Yao [6] and Capinski et al. [7] reported a similar trend in the thermal conductivity of GaAs/AlAs superlattices.

Recent progress in the chemical vapor deposition (CVD) $[11,12]$ technique supports the fabrication of high-purity diamond thin films by controlling their isotope ratio. Watanabe

\footnotetext{
*ogi@prec.eng.osaka-u.ac.jp
}

et al. [12] discovered that ${ }^{12} \mathrm{C} /{ }^{13} \mathrm{C}$ superlattice diamond thin films exhibit special electrical properties in cathodoluminescence, which cannot be explained by individual monolayer characteristics: The excitonic recombination in the ${ }^{13} \mathrm{C}$ diamond layer disappears even though ${ }^{13} \mathrm{C}$ diamond is involved. Such a specific property of the ${ }^{12} \mathrm{C} /{ }^{13} \mathrm{C}$ superlattice will appear in phonon properties like the elastic constant and thermal conductivity. Furthermore, since isotopically pure diamond exhibits extremely high thermal conductivity even at room temperature $\left(\sim 3000 \mathrm{~W} \mathrm{~m}^{-1} \mathrm{~K}^{-1}\right)$ [13], controlling the thermal conductivity of a diamond thin film in a wide range using superlatticing is possible. However, no experimental reports on phonon properties of the ${ }^{12} \mathrm{C} /{ }^{13} \mathrm{C}$ superlattice exist.

This study evaluates the elastic constant and the thermal conductivity of epitaxially grown ${ }^{12} \mathrm{C} /{ }^{13} \mathrm{C}$ diamond superlattices with different layer thicknesses and proposes a lattice-dynamics calculation to explain the measurements of thermal conductivity. Because the lattice mismatch between each layer $(\sim 0.01 \%)$ is smaller than those in traditional superlattices, we expect defect-free interfaces, allowing us to compare measurements and theory without the ambiguity caused by the defects.

\section{EXPERIMENTS}

We synthesized [100] homoepitaxial isotope-controlled diamond and isotopic diamond superlattice thin films on a [100] diamond substrate $\left(3 \times 3 \times 0.5 \mathrm{~mm}^{3}\right)$ with a natural isotope content $\left(98.9 \%{ }^{12} \mathrm{C}\right.$ and $\left.1.1 \%{ }^{13} \mathrm{C}\right)$ using the microwave plasma-assisted CVD method [11,12]. The isotope purity of ${ }^{12} \mathrm{CH}_{4}$ and ${ }^{13} \mathrm{CH}_{4}$ methane gases were $99.999 \%$ and $99 \%$, respectively. We prepared two monolayer isotope diamonds 
with $99.999 \%{ }^{12} \mathrm{C}$ and $99 \%{ }^{13} \mathrm{C}$, referred to as ${ }^{12} \mathrm{C}$ and ${ }^{13} \mathrm{C}$, respectively. (These concentrations indicate the isotope purity, not the chemical purity. The chemical purity exceeds $99.99 \%$ in our specimens.) We also prepared three isotopic diamond superlattices composed of ${ }^{12} \mathrm{C}$ and ${ }^{13} \mathrm{C}$ diamond layers of the same thickness. Their layer thicknesses are 1,30, and $100 \mathrm{~nm}$, and these specimens are referred to as SL1, SL30, and SL100, respectively. The total film thickness of all specimens is about $3 \mu \mathrm{m}$. A 10-nm Pt thin film was then deposited on the surface of each specimen using the magnetron sputtering method as the source of ultrasonic waves.

Picosecond ultrasound spectroscopy [14-17] was employed in this study. A Ti:sapphire pulse laser with 140-fs duration and $80-\mathrm{MHz}$ repetition rate was used, and the light pulse was divided into the pump and probe lights. Their wavelengths were 800 and $400 \mathrm{~nm}$, respectively. We irradiated the Pt thin film with the pump light pulse to generate the ultrahigh frequency $(\lesssim 1 \mathrm{THz})$ coherent ultrasonic pulse via transient thermal stress in the Pt film. The probe light was then focused on the surface, which passed through the Pt film and was diffracted by the ultrasonic pulse through the photoelastic effect. The light path of the pump light pulse was controlled using a mechanical delay. The total reflectivity changes periodically due to the moving ultrasonic pulse, causing backward diffraction of the probe light. From this oscillation (Brillouin oscillation) frequency, the acoustic-wave speed and the elastic constant are determined [18-20]. From the photothermal effect [15], the surface temperature change is proportional to the change in probe light reflectivity, from which we evaluated the thermal conductivity. The details of our optical system, the calculation of the acoustic-wave speed, and elasticity are shown elsewhere [20-23]. Just after the excitation, however, the reflectivity change is highly affected by the electron thermalization process, and we adopt a reference sample in this paper to compensate this effect.

\section{THEORETICAL CALCULATION}

The lattice thermal conductivity calculation starts from phonon kinetic theory:

$$
\kappa=\sum_{\lambda} \kappa_{\lambda}=\sum_{\lambda} \tau_{\lambda} C(\omega) v_{\lambda, z}^{2}
$$

where $\lambda$ denotes the phonon mode, depending on the wave number $\left(\mathbf{k}_{\|}, k_{z}\right)$, frequency $\omega$, and polarization. The total thermal conductivity $\kappa$ is given by the summation of contributions of different phonon modes $\kappa_{\lambda}$. The specific heat $C(\omega)$ can be calculated using a quantum harmonic oscillator:

$$
C(\omega)=\frac{\partial}{\partial T}\left[\hbar \omega\left(N_{0}+1 / 2\right)\right],
$$

where $N_{0}$ denotes the Planck distribution function. The outof-plane group velocity $v_{\lambda, z}$ can be calculated using lattice dynamics even for a large system as follows. The phonon mean free time $\tau_{\lambda}$ is not straightforward to estimate, and we first assume it to be constant at each mode and separately investigate the effect of the group-velocity distribution by calculating the normalized thermal conductivity $\tilde{\kappa}$ using the phonon mean free time. This normalization using phonon mean free time was proposed by Tamura et al. [24]. They

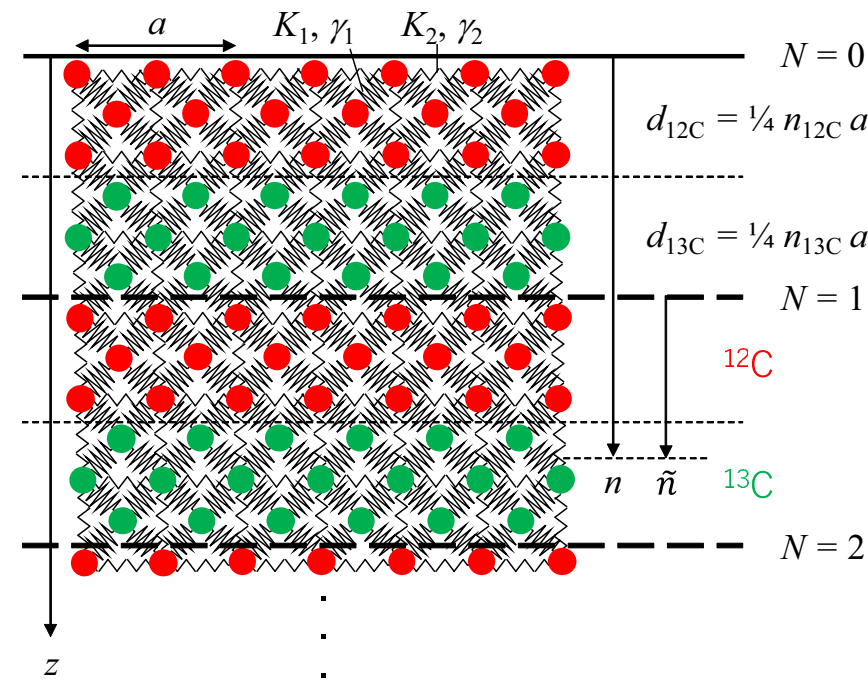

FIG. 1. Schematic of the side view (not cross section) of the $(3,3)$ diamond superlattice. $N$ denotes the number of superlattice periods from the global origin, and $\tilde{n}$ is the layer number from the subunit origin. $n=N\left(n_{12 C}+n_{13 C}\right)+\tilde{n}$ is the total layer number from the global origin.

further simplified the diamond structure crystals to one fcc lattice, including only one force-constant parameter. However, we adopt a more complete model. Pandey and Dayal $[25,26]$ indicated that the four-constant model gives better agreement between experimental and theoretical phonon dispersions. Our model connects each atom to the four first-nearestneighbor atoms using the bond-stretching and bond-bending connections with constants $K_{1}$ and $\gamma_{1}$, respectively. It also connects 12 second-nearest-neighbor atoms using the bondstretching connections with constant $K_{2}$ and the bond-bending constant $\gamma_{2}$ (Fig. 1). These values will be determined using experimental data. Those from Warren et al.'s [27] data based on inelastic neutron scattering are given in Table I. From the calculation of lattice dynamics, described later, we derived the expressions for $\mathrm{LO}(\Gamma), \mathrm{LO}(X), \mathrm{TA}(X)$, and $\mathrm{TO}(X)$ as follows:

$$
\begin{gathered}
M \omega_{\mathrm{LO}}^{2}(\Gamma)=\frac{8}{3}\left(K_{1}+2 \gamma_{1}\right), \\
M \omega_{\mathrm{LO}}^{2}(X)=\frac{4}{3}\left(K_{1}+2 \gamma_{1}\right)+8\left(K_{2}+\gamma_{2}\right), \\
M \omega_{\mathrm{TA}}^{2}(X)=8 K_{2}+4 \gamma_{1}, \\
M \omega_{\mathrm{TO}}^{2}(X)=\frac{4}{3}\left(2 K_{1}+\gamma_{1}\right)+8 K_{2},
\end{gathered}
$$

where $M$ denotes the atomic mass of the ${ }^{12} \mathrm{C}$ diamond. $\mathrm{Be}$ cause the sound velocity from Warren et al.'s experiment slightly differs from our measurement for the ${ }^{12} \mathrm{C}$ diamond

TABLE I. Phonon frequencies $(\mathrm{THz})$ and force constants for bond stretching and bond bending $\left(\mathrm{N} \mathrm{m}^{-1}\right)$ used in this study.

\begin{tabular}{lcccccccc}
\hline \hline & $\frac{\omega_{\mathrm{LO}}}{2 \pi}(\Gamma)$ & $\frac{\omega_{\mathrm{LO}}}{2 \pi}(X)$ & $\frac{\omega_{\mathrm{TO}}}{2 \pi}(X)$ & $\frac{\omega_{\mathrm{TA}}}{2 \pi}(X)$ & $K_{1}$ & $\gamma_{1}$ & $K_{2}$ & $\gamma_{2}$ \\
\hline Ref. [27] & 40.0 & 35.5 & 32.1 & 23.0 & 278.0 & 116.4 & -1.9 & 51.1 \\
Present study & 40.0 & 36.5 & 33.0 & 26.0 & 238.4 & 116.5 & 8.2 & 44.2
\end{tabular}


TABLE II. The calculated and measured longitudinal-wave velocities $v_{l}^{\text {cal }}$ and $v_{l}^{\text {mea }}(\mathrm{m} / \mathrm{s})$ and measured out-of-plane longitudinal elastic constant $C_{\perp}^{\text {mea }}(\mathrm{GPa})$. The error range was calculated from the standard deviation of more than 10 measurements on different points of each specimen.

\begin{tabular}{lccccc}
\hline \hline & ${ }^{12} \mathrm{C}$ & ${ }^{13} \mathrm{C}$ & SL1 & SL30 & SL100 \\
\hline$v_{l}^{\text {cal }}$ & 17109 & 16436 & 16764 & 16764 & 16764 \\
$v_{l}^{\text {mea }}$ & $17492 \pm 3$ & $16806 \pm 3$ & $17213 \pm 42$ & $17164 \pm 9$ & $17168 \pm 12$ \\
$C_{\perp}^{\text {mea }}$ & $1074.6 \pm 0.3$ & $1074.5 \pm 0.4$ & $1081.5 \pm 4.1$ & $1077.8 \pm 1.2$ & $1078.2 \pm 1.6$ \\
\hline \hline
\end{tabular}

specimen, we used force constants different from the values from their measurements, as shown in Table I. The forceconstant values for ${ }^{13} \mathrm{C}$ are considered the same as those of ${ }^{12} \mathrm{C}$ because the isotopic effect on elastic constants in diamonds is negligible [28].

Figure 1 illustrates the simulation model. We define $n_{12 C}$ as the number of ${ }^{12} \mathrm{C}$ diamond layers in the unit and $n_{13 C}$ as that of the ${ }^{13} \mathrm{C}$ diamond layers. $d_{12 C}$ and $d_{13 C}$ denote the sublattice thicknesses, respectively, so that $D=d_{12 C}+d_{13 C}$ corresponds to the superlattice period. We refer to $\left(n_{12 C}, n_{13 C}\right)$ as a superlattice composed of $n_{12 C}{ }^{12} \mathrm{C}$ and $n_{13 C}{ }^{13} \mathrm{C}$ diamond layers. $a$ denotes the lattice constant of pure ${ }^{12} \mathrm{C}$ or ${ }^{13} \mathrm{C}$ diamond, and its value is $3.5671 \AA$ [29]. The equation of atomic motion follows from Newton's second law:

$$
F_{\alpha}(i, j)=M_{i} \ddot{u}_{\alpha}(i, j)=-\sum_{i^{\prime}, j^{\prime}, \beta} \Phi_{\alpha \beta}^{i j, i^{\prime} j^{\prime}} u_{\beta}\left(i^{\prime}, j^{\prime}\right),
$$

where $\alpha$ and $\beta$ indicate the direction, $(i, j)$ denote the numbers of atoms in the cell, and $\Phi$ is the force-constant matrix [30] expressed by the bond-stretching and bond-bending force constants. The equilibrium position is defined as $\mathbf{r}_{l m n}=$ $\left(\mathbf{x}_{l m}, z_{n}\right)=(l, m, n) a / 4$. From Bloch's theorem [24,31], the displacement $\mathbf{u}_{l m n}$ can be expressed as

$$
\mathbf{u}_{l m n}=\mathbf{u}_{\tilde{n}} \exp \left[i\left(\mathbf{k}_{\|} \cdot \mathbf{x}_{l m}+k_{z} N D-\omega t\right)\right],
$$

where $N$ indicates the number of periods (Fig. 1). Substituting Eq. (8) into Eq. (7) and setting $u_{\alpha}(i)=1 / \sqrt{M_{i}} e_{\alpha}(i)$, where $e_{\alpha}(i)$ denotes the $\alpha$ component of the unit vector of the $i$ th atom, we have

$$
\omega^{2} e_{\alpha}(i)=\sum_{i^{\prime}, \beta} D_{\alpha \beta}\left(i, i^{\prime}\right) e_{\beta}\left(i^{\prime}\right),
$$

where

$$
D_{\alpha \beta}\left(i, i^{\prime}\right)=\frac{1}{\sqrt{M_{i} M_{i^{\prime}}}} \sum_{i^{\prime}, j^{\prime}, \beta} \Phi_{\alpha \beta}^{i j, i^{\prime} j^{\prime}} \exp \left[i\left(\mathbf{k}_{\|} \cdot \mathbf{x}_{l m}+k_{z} N D\right)\right]
$$

is the dynamical matrix. The dispersion relation and phonon density of state (PDOS) can then be obtained by solving the eigenvalue problem in Eq. (9).

\section{RESULTS AND DISCUSSION}

Figure 2(a) illustrates the as-measured reflectivity change in two specimens. It instantly rises at the zero point because of the transient heat supplied by the pump light pulse, quickly drops due to electron thermalization and the energy transfer to phonons, gradually attenuates due to thermal diffusion, and vibrates due to Brillouin oscillation. We first removed the baseline attenuation to obtain Brillouin oscillation [Fig. 2(b)] and used fast Fourier transform to determine the Brillouin-oscillation frequency [Fig. 2(e)]. The longitudinal-wave velocity and the out-of-plane elastic constant are determined using the Brillouin-oscillation frequency, the wavelength of the probe light, the refractive index [32,33], and the mass density, as shown in Table II. Note that the isotope diamond superlattices exhibit very large elastic constants, which are comparable to (or even higher than) those of pure isotope diamonds. Since the out-of-plane elastic constant of a superlattice is significantly smaller than that estimated from each layer [8-10], the high elasticity of the isotope diamond superlattice is surprising, and also it confirms that there are almost no interfacial defects in our specimens, allowing the comparison between the measured and calculated thermal conductivities.

We subtracted the Brillouin oscillation from the change in reflectivity to find the change caused by the surface temperature change and normalized it using the maximum reflectivity change at the zero point [Fig. 2(c)]. The transient increase in reflectivity at the zero point reflects electron excitation, and the subsequent drop is mainly caused by the electronthermalization process in the Pt layer, during which the phonon temperature rises through the energy transfer from excited electrons to lattices $[34,35]$. As shown in inset in Fig. 2(d), the reflectivity after $\sim 0.5 \mathrm{ps}$ is affected by the thermal conductivity of the material under the Pt layer, indicating that it will be possible to evaluate the thermal conductivity from the reflectivity change after the drop by comparing thermal conduction theory to experiments [7]. We used the one-dimensional thermal conduction model in a three-layer system, consisting of the top Pt layer, the middle diamond specimen layer, and the bottom substrate layer, to calculate the surface temperature change after transient heating [21]. This one-dimensional model applies because the diameter of the laser beam is about $10 \mu \mathrm{m}$, which is much larger than the thermal-phonon propagation distance of $\sim 400 \mathrm{~nm}$ within $200 \mathrm{ps}$ in diamond. However, there are two ambiguities in this evaluation. First, it is difficult to estimate the temperature increase at the top surface in the experiment because of the complexity in the electron-phonon thermalization process. Second, the thermal conductance between the Pt layer and diamond specimen is highly ambiguous $[15,36]$. Therefore, we used two reference specimens to minimize these effects on the estimation of the thermal conductivity as follows. The simulated surface temperature can be determined by the thermal conductivity of the specimen, interfacial conductance between $\mathrm{Pt}$ and the specimen, and the surface temperature at the zero point as well as other known properties. We have first evaluated the apparent surface temperature at the zero 

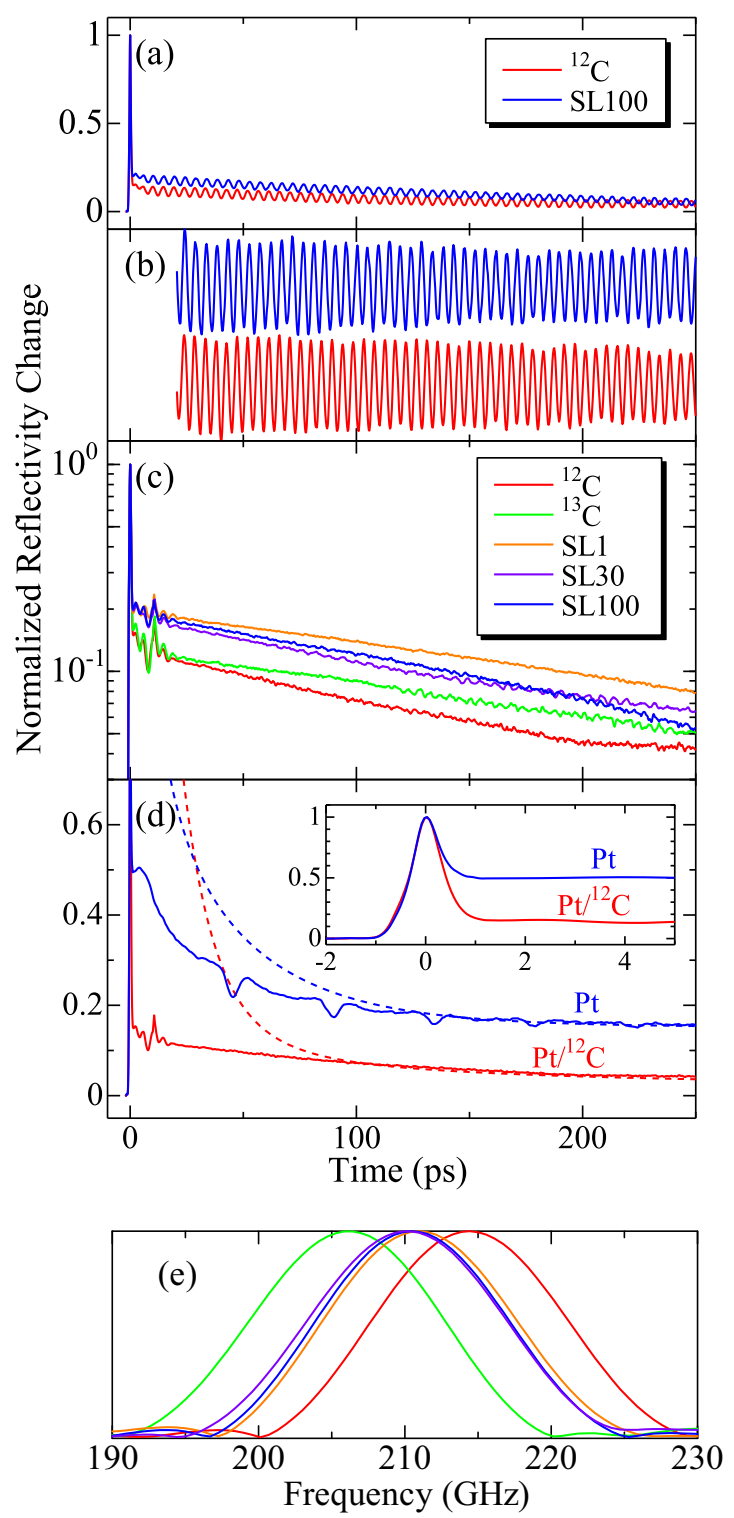

FIG. 2. Picosecond ultrasound spectroscopy measurements. (a) As-measured probe light reflectivity of ${ }^{12} \mathrm{C}$ and SL100 thin films. (b) Brillouin oscillations after removing the background attenuation. (c) Thermal cooling-down behavior after removing Brillouin oscillations for the five specimens. (d) As-measured probe light reflectivity for the 93-nm Pt film and the ${ }^{12} \mathrm{C}$ specimen (solid curves) and calculated surface-temperature changes (dashed lines). The inset shows the measured reflectivity changes near the zero point. Small reflectivity drops with a $\sim 45$-ps interval observed in the Pt specimen indicate the pulse-echo signals of the coherent phonon. (e) Fast Fourier transform spectra of Brillouin oscillations for the five specimens.

point using a 93-nm Pt monolayer specimen as the reference specimen, which was deposited under the same deposition condition as the Pt films on the diamond specimens. By fitting the simulated temperature change to the experiment, we evaluated the zero-point surface temperature [Fig. 2(d)]. In this estimation, the surface temperature of the 10-nm Pt film on the diamond specimens would be underestimated because part of the light pulse would pass through the Pt layer. However, considering the absorption coefficient $\left(1.27 \times 10^{8} \mathrm{~m}^{-1}\right)$ of the pump light $(800 \mathrm{~nm})$ on $\mathrm{Pt}$, the penetration depth will be smaller than $10 \mathrm{~nm}$, and a large amount of energy of the pump light is absorbed in the Pt layer, while the penetration depth of the probe light $(400 \mathrm{~nm})$ is slightly larger than $10 \mathrm{~nm}$, producing the Brillouin oscillation. Therefore, the effect of underestimation of the surface temperature increase caused by the pump light will be insignificant.

Next, we estimated the interface conductance by fitting the simulated temperature change to the experiment using the ${ }^{12} \mathrm{C}$ diamond specimen as the other reference, where the zero-point temperature value evaluated from the Pt reference specimen was used. (The thermal conductivity of $99.93 \%{ }^{12} \mathrm{C}$ is reported to be $3320 \mathrm{~W} \mathrm{~m}^{-1} \mathrm{~K}^{-1}$ [13], and we used this value for the reference material.) The interface conductance value estimated was $2.55 \times 10^{9} \mathrm{~W} \mathrm{~m}^{-2} \mathrm{~K}^{-1}$, which is much larger than that estimated between Pd and diamond [15]. One reason is the ambiguity of the surface temperature evaluation using the thick Pt monolayer specimen as indicated above. Such ambiguity is, however, involved in the estimation of the apparent interface conductance using the ${ }^{12} \mathrm{C}$ reference specimen. Another reason is that the interface conductance in our specimen is actually significantly larger than that in the previous study [15]. For example, the normalized reflectivity at $200 \mathrm{ps}$ in the previous study is higher than that in our ${ }^{12} \mathrm{C}$ diamond specimen by a factor of 12 , indicating very low heat resistance at the interface in our specimen.

Finally, thermal conductivities of other specimens are evaluated by fitting the simulated temperature changes to the experiments using thus estimated parameters. We used reflectivity changes for this evaluation between 100 and 190 ps.

Figure 3(a) presents the calculated dispersion curves. The frequency gap is observed at the folded Brillouin-zone boundary for a superlattice due to the difference in mass between ${ }^{12} \mathrm{C}$ and ${ }^{13} \mathrm{C}$. The longitudinal-wave speed $v_{l}$ is calculated using the linear zone, as shown in Table II. The trend of the calculated longitudinal-wave speed is equivalent to that of the measurements. Figure 3(b) presents the PDOS. The curve saturates as $n \gtrsim 60$. Evaluating the effect of the outof-plane phonon group velocity on the thermal conduction, the weighted group phonon velocity, calculated using the out-of-plane phonon group velocity and the PDOS, is used [24], as shown in Fig. 3(c). The weighted distribution drops significantly (by $\sim 30 \%$ ) in the superlattice structures.

Figure $3(\mathrm{~d})$ shows the calculated thermal conductivity normalized using the relaxation time $\tilde{\kappa}$. The isotopic diamond superlattices show thermal conductivities lower than those of pure isotope diamonds by $\sim 40 \%$. This deterioration in thermal conduction is principally due to the decrease in the phonon group velocity [Fig. 3(c)]. We note that $\tilde{\kappa}$ of superlattice diamond decreases with increased superlattice period because the longer the superlattice period is, the more folds there are at the Brillouin zone, which results in a larger decrease in the phonon group velocity.

Table III compares the measured and calculated thermal conductivities. The phonon mean free time takes the same value in all specimens and all phonon modes, determined by measuring the reference specimen of ${ }^{12} \mathrm{C}$ diamond. The measured thermal conductivity of ${ }^{13} \mathrm{C}$ diamond is lower than the calculated value by $50 \%$, and we attribute this to the reduction 
(a)
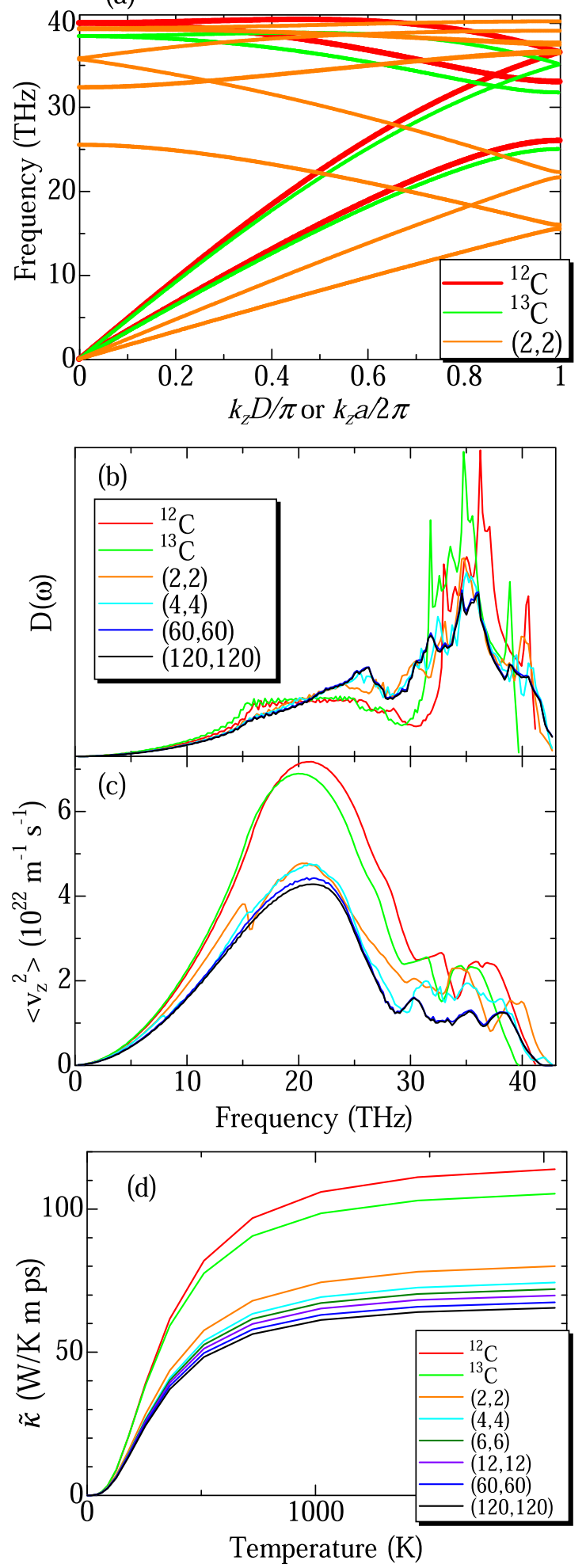

FIG. 3. Calculation results of (a) phonon dispersion curves of ${ }^{12} \mathrm{C},{ }^{13} \mathrm{C}$, and $(2,2)$ diamonds, (b) PDOS, (c) DOS weighted by the square out-of-plane-component velocity, and (d) lattice thermal conductivity normalized by the relaxation time $\tilde{\kappa}$ for various superlattice periods $\left(n_{12 C}, n_{13 C}\right)$.

of the mean free time due to scattering by isotope impurities. Such an isotope impurity effect on the thermal conductivity
TABLE III. The lattice thermal conductivity $\tilde{\kappa}$ normalized by phonon mean free time $\left(\mathrm{W} \mathrm{m}^{-1} \mathrm{~K}^{-1} \mathrm{ps}^{-1}\right)$, the corresponding thermal conductivity $\tilde{\kappa} \times \tau(\tau=68.2 \mathrm{ps})$, and measured thermal conductivity $\kappa\left(\mathrm{W} \mathrm{m}^{-1} \mathrm{~K}^{-1}\right)$ at $297 \mathrm{~K}$. The $\tau$ value was determined using the experimental ${ }^{12} \mathrm{C}$ diamond value. SL1 is equivalent to $(12,12)$ and SL30 and SL100 are treated to be the same as $(336,336)$.

\begin{tabular}{lccccc}
\hline \hline & ${ }^{12} \mathrm{C}$ & ${ }^{13} \mathrm{C}$ & SL1 & SL30 & SL100 \\
\hline$\tilde{\kappa}$ & 48.7 & 47.3 & 32.3 & 29.0 & 29.0 \\
$\tilde{\kappa} \times \tau$ & 3320 & 3229 & 2163 & 1980 & 1980
\end{tabular}

Measured $3320 \pm 7971687 \pm 471708 \pm 921211 \pm 2821209 \pm 284$

was measured by Anthony et al. [13], Onn et al. [37], and Olson et al. [38]. These studies indicated that even $1 \%$ impurity decreased the thermal conductivity by more than $\sim 30 \%$. Since our ${ }^{13} \mathrm{C}$ diamond also contains $\sim 1 \%$ isotope impurity, the measured thermal conductivity is smaller than the calculated (ideal) value. Because the superlattices contain ${ }^{13} \mathrm{C}$ diamond with a volume fraction of $50 \%$, we expect an additional $25 \%$ reduction in the thermal conductivity due to the isotope effect. However, even involving this effect, the measurements for the superlattices are smaller than the theoretical estimations. This discrepancy appears most remarkably in specimen SL1. Therefore, we need to further consider a mechanism that is specific to the superlattice to affect the mean free time.

We consider the mini-umklapp scattering [39] a specifically effective explanation for the isotope diamond superlattice, as discussed later. Ren and Dow [39] proposed the mini-umklapp scattering model, which describes the threephonon umklapp scattering in a superlattice. The frequency gap at the folded Brillouin zone causes the change in the umklapp scattering. The scattering rate takes the form

$$
\begin{gathered}
\tau_{U}^{-1}=B_{U} T^{3} \omega^{2}+c / L, \\
\tau_{N}^{-1}=B_{N} T^{3} \omega^{2},
\end{gathered}
$$

where $c$ represents the sound velocity and $L$ denotes the characteristic size. Since the second term in Eq. (11) is negligible compared with the first term at room temperature [40], we

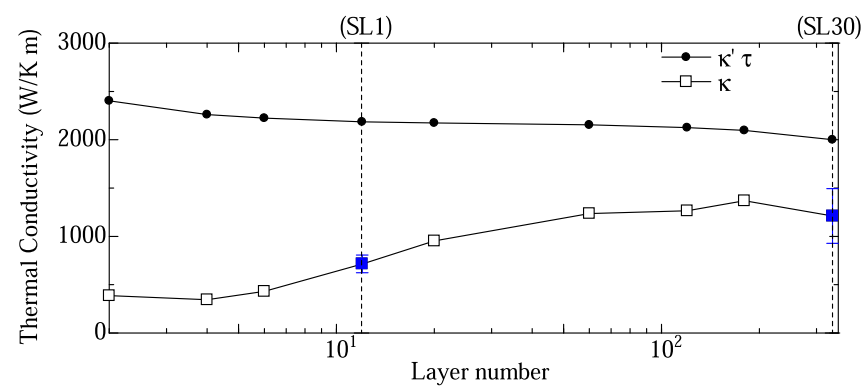

FIG. 4. Lattice thermal conductivity calculated using a constant relaxation time (solid circles) and that involving the mini-umklapp scattering contribution (open squares) for various layer numbers $\left(n_{12 C}=n_{13 C}\right)$ at $297 \mathrm{~K}$. We assume $C_{U}=9.63 \times 10^{-20} \mathrm{~s} \mathrm{~K}^{-3}, s=$ $0.646, \Theta=2230 \mathrm{~K}$, and $c / L=0$. The blue squares indicate the experimental data used to obtain the parameters $C_{U}$ and $s$. 


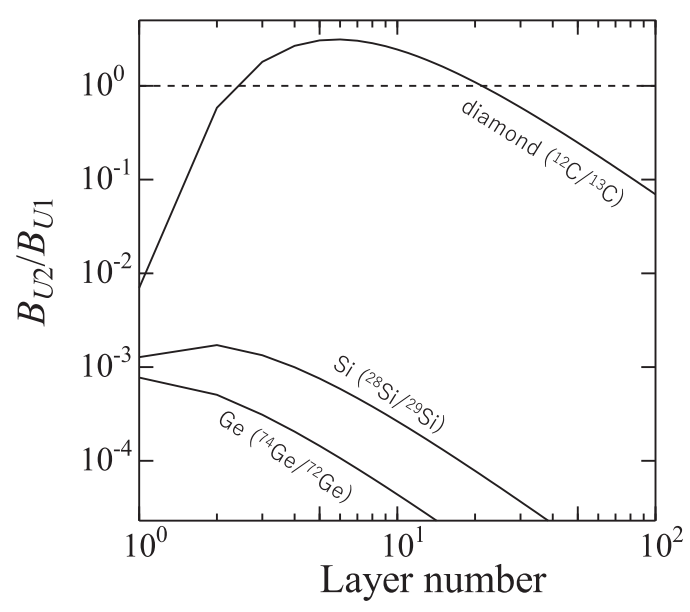

FIG. 5. The contribution of the mini-umklapp scattering effect in three isotope superlattices at $297 \mathrm{~K}$.

ignored the $c / L$ term. The coefficient $B_{U}$ takes the form

$$
B_{U}=C_{U}\left\{e^{-\frac{\Theta}{s T}}+\frac{1}{\left(n_{1}+n_{2}\right)^{2}}\left(\frac{\Delta M}{M}\right)^{2} e^{-\frac{\Theta}{s\left(n_{1}+n_{2}\right) T}}\right\},
$$

where $\Theta$ is the Debye temperature and $C_{U}$ and $s$ are constants. $\Delta M / M$ denotes the fractional mass difference, and $n_{1}$ and $n_{2}$ are the numbers of atomic layers of ${ }^{12} \mathrm{C}$ and ${ }^{13} \mathrm{C}$ diamonds, respectively. The first and second terms in Eq. (13) indicate Callaway [40] and mini-umklapp scattering contributions, respectively. $\Theta$ is given as $2230 \mathrm{~K}$ for diamond [41]. Because separation between these terms is never straightforward, Ren and Dow [39] assumed that the mini-umklapp processes would dominate the thermal resistance in a superlattice, and they simply took $B_{U}+B_{N}=B_{U}$. We follow this simplification and combine the $B_{N}$ term with the $B_{U}$ term. We thus find the appropriate $C_{U}$ and $s$ values using the measured thermal conductivity data of SL1 $(12,12)$ and SL30 $(336,336)$. Figure 4 shows the calculated result with open symbol. The thermal conductivity increases between 1 and 100 layers, where the mini-umklapp scattering becomes significant, and saturates for larger layer numbers because of the decrease in the phonon group velocity.

Importantly, we note that the mini-umklapp scattering effect [the second term in Eq. (13)] in an isotopic superlattice is considerable only for diamond at room temperature, as shown in Fig. 5, where the ratio of the second term $\left(B_{U 2}\right)$ to the first term $\left(B_{U 1}\right)$ in Eq. (13) is examined for some isotope superlattices. The mini-umklapp scattering effect is dominant only for diamond superlattices with layer numbers between 2 and 20. This follows for two reasons: First, diamond has extremely high Debye temperature; thereby, room temperature is apparently a low temperature for diamond. Second, diamond has a large fractional isotope mass difference because of its light atomic mass. Thus, diamond is a rare material, where the mini-umklapp scattering effect is pronounced in isotope superlatticing.

\section{CONCLUSION}

We measured the elastic constant and thermal conductivity of isotopic superlattice diamond thin films using ultrafast pump-probe laser measurement and developed a theoretical calculation for the lattice thermal conductivity. The out-ofplane longitudinal-wave elastic constant of the superlattice remained very large despite the many interfaces involved. This result indicated that superlattices that are almost defect free were successfully fabricated in this study. The thermal conductivity of isotopic superlattice diamond can be lowered by $\sim 40 \%$ from that of the high-purity diamond because of the decrease in the out-of-plane phonon group velocity even without interfacial defects. The trend of the calculated lattice thermal conductivity agreed with that of the measurements, but the superlattice with 1-nm sublattice thickness showed a large difference between them. As a possible scattering mechanism, we incorporated the mini-umklapp three-phonon effect into our model to explain the measurements. The mini-umklapp effect normally becomes significant only at low temperatures and negligible near room temperature. However, our results showed that this effect can be dominant in an isotope diamond superlattice even at room temperature because of the extremely high Debye temperature and large mass difference.

The data that support the findings of this study are available from the corresponding author upon request.

\section{ACKNOWLEDGMENT}

This study was supported by JSPS KAKENHI Grant No. $19 \mathrm{H} 00862$.
[1] S. Froyen, D. M. Wood, and A. Zunger, Phys. Rev. B 37, 6893 (1988).

[2] D. Menzel, W. Koschinski, K. Dettmer, and J. Schoenes, Thin Solid Films 342, 312 (1999).

[3] H. Sakaki, T. Noda, K. Hirakawa, M. Tanaka, and T. Matsusue, Appl. Phys. Lett. 51, 1934 (1987).

[4] M. N. Baibich, J. M. Broto, A. Fert, F. Nguyen Van Dau, F. Petroff, P. Etienne, G. Creuzet, A. Friederich, and J. Chazelas, Phys. Rev. Lett. 61, 2472 (1988).

[5] S. M. Lee, D. G. Cahill, and R. Venkatasubramanian, Appl. Phys. Lett. 70, 2957 (1997).

[6] T. Yao, Appl. Phys. Lett. 51, 1798 (1987).
[7] W. S. Capinski, H. J. Maris, T. Ruf, M. Cardona, K. Ploog, and D. S. Katzer, Phys. Rev. B 59, 8105 (1999).

[8] N. Nakamura, H. Ogi, T. Yasui, M. Fujii, and M. Hirao, Phys. Rev. Lett. 99, 035502 (2007).

[9] N. Nakamura, A. Uranishi, M. Wakita, H. Ogi, and M. Hirao, Jpn. J. Appl. Phys. 49, 07HB04 (2010).

[10] N. Nakamura, R. Yokomura, N. Takeuchi, D. Yamakado, and H. Ogi, Jpn. J. Appl. Phys. 58, 075504 (2019).

[11] M. C. Costello, D. A. Tossell, D. M. Reece, and C. J. Brierley, Diamond Relat. Mater. 3, 1137 (1994).

[12] H. Watanabe, C. E. Nebel, and S. Shikata, Science 324, 1425 (2009). 
[13] T. R. Anthony, W. F. Banholzer, J. F. Fleischer, L. Wei, P. K. Kuo, R. L. Thomas, and R. W. Pryor, Phys. Rev. B 42, 1104 (1990).

[14] C. Thomsen, J. Strait, Z. Vardeny, H. J. Maris, J. Tauc, and J. J. Hauser, Phys. Rev. Lett. 53, 989 (1984).

[15] R. J. Stoner and H. J. Maris, Phys. Rev. B 48, 16373 (1993).

[16] C. Thomsen, H. T. Grahn, H. J. Maris, and J. Tauc, Phys. Rev. B 34, 4129 (1986).

[17] O. Matsuda, O. B. Wright, D. H. Hurley, V. E. Gusev, and K. Shimizu, Phys. Rev. Lett. 93, 095501 (2004).

[18] A. Devos and R. Cote, Phys. Rev. B 70, 125208 (2004).

[19] H. Ogi, T. Shagawa, N. Nakamura, M. Hirao, H. Odaka, and N. Kihara, Phys. Rev. B 78, 134204 (2008).

[20] H. Ogi, T. Shagawa, N. Nakamura, M. Hirao, H. Odaka, and N. Kihara, Jpn. J. Appl. Phys. 48, 07GA01 (2009).

[21] H. K. Weng, A. Nagakubo, H. Watanabe, and H. Ogi, Jpn. J. Appl. Phys. 59, SKKA04 (2020).

[22] A. Nagakubo, S. Tsuboi, Y. Kabe, S. Matsuda, A. Koreeda, Y. Fujii, and H. Ogi, Appl. Phys. Lett. 114, 251905 (2019).

[23] H. Ogi, S. Iwagami, A. Nagakubo, T. Taniguchi, and T. Ono, Sensors Actuators, B 278, 15 (2019).

[24] S. I. Tamura, Y. Tanaka, and H. J. Maris, Phys. Rev. B 60, 2627 (1999).

[25] B. P. Pandey and B. Dayal, Solid State Commun. 11, 775 (1972).

[26] B. P. Pandey and B. Dayal, J. Phys. C 6, 2943 (1973).

[27] J. L. Warren, J. L. Yarnell, G. Dolling, and R. A. Cowley, Phys. Rev. 158, 805 (1967).
[28] R. Vogelgesang, A. K. Ramdas, S. Rodriguez, M. Grimsditch, and T. R. Anthony, Phys. Rev. B 54, 3989 (1996).

[29] T. Yamanaka, S. Morimoto, and H. Kanda, Phys. Rev. B 49, 9341 (1994).

[30] R. G. Leisure, Ultrasonic Spectroscopy: Application in Condensed Matter Physics and Material Science (Cambridge University Press, Cambridge, 2017), Chap. 3-2.

[31] A. A. Maradudin, P. A. Flinn, and R. A. Coldwell-Horsfall, Ann. Phys. (NY) 15, 360 (1961).

[32] T. Ruf, M. Cardona, C. S. J. Pickles, and R. Sussmann, Phys. Rev. B 62, 16578 (2000).

[33] E. D. Palik, Handbook of Optical Constants of Solids, Vol. 1. (Academic, San Diego, 1985), p. 672.

[34] G. Tas and H. J. Maris, Phys. Rev. B 49, 15046 (1994).

[35] A. Block, M. Liebel, R. Yu, M. Spector, Y. Sivan, F. J. Garcia De Abajo, and N. F. Van Hulst, Sci. Adv. 5, eaav8965 (2019).

[36] A. J. Schmidt, X. Chen, and G. Chen, Rev. Sci. Instrum. 79, 114902 (2008).

[37] D. G. Onn, A. Witek, Y. Z. Qiu, T. R. Anthony, and W. F. Banholzer, Phys. Rev. Lett. 68, 2806 (1992).

[38] J. R. Olson, R. O. Pohl, J. W. Vandersande, A. Zoltan, T. R. Anthony, and W. F. Banholzer, Phys. Rev. B 47, 14850 (1993).

[39] S. Y. Ren and J. D. Dow, Phys. Rev B 25, 3750 (1982).

[40] J. Callaway, Phys. Rev. 113, 1046 (1959).

[41] C. Kittel, Introduction to Solid State Physics (Wiley, Hoboken, NJ, 2004), Chap. 5. 